\title{
DAMP-driven metabolic adaptation
}

the production
of pro-IL-1 $\beta$
was markedly
increased in
cells exposed
to LPS and
oxPAPC ...
compared with
LPS only

Pathogen-associated molecular patterns such as lipopolysaccharide (LPS) both induce the expression of inflammatory cytokines such as IL-1 $\beta$ and lead to changes in macrophage metabolism that involve a shift from mitochondrial oxidative phosphorylation (OXPHOS) to aerobic glycolysis. While it is known that endogenous damage-associated molecular patterns (DAMPs), such as oxidized phospholipids, can similarly induce inflammatory gene expression and inflammasome activation, it has not been known whether they also drive metabolic adaptation. A new study in Nature Immunology shows that a class of oxidized phospholipids known as oxPAPC can indeed induce a unique 'hypermetabolic' state in macrophages.

When bone marrow-derived macrophages were primed with LPS and then exposed to oxPAPC, glycolysis was increased but without the accompanying decrease in OXPHOS that occurs in response to LPS alone. This hypermetabolic state of simultaneous OXPHOS

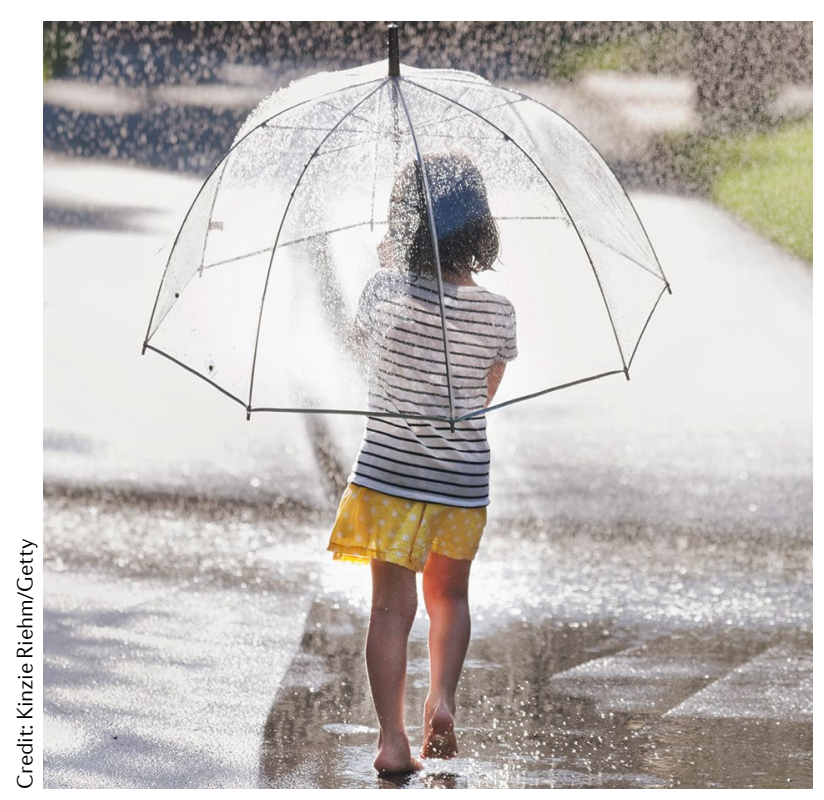

and aerobic glycolysis could not be induced by exposure to LPS or oxPAPC alone.

In keeping with the known influence of macrophage metabolism on inflammatory cytokine production, the production of pro-IL-1 $\beta$ was markedly increased in cells exposed to LPS and oxPAPC (co-treated cells) compared with LPS only. Further studies showed that inhibiting OXPHOS decreased the capacity of the hypermetabolic macrophages to produce IL- $1 \beta$. OXPHOS is normally inhibited in cells exposed to LPS through the production of nitric oxide, but the authors showed that oxPAPC administration to LPStreated macrophages inhibits nitric oxide production in a dose-dependent manner by decreasing levels of inducible nitric oxide synthase. In addition to OXPHOS, glutaminolysis was also required for the increased production of IL- $1 \beta$ by co-treated macrophages, and oxPAPC was shown to increase the transcription of several genes involved in glutamine transport and metabolism. Thus, oxPAPC promotes OXPHOS and stimulates glutamine metabolism, which together contribute to a hyperinflammatory state.

Levels of the metabolite-sensitive transcription factor hypoxiainducible factor $1 \alpha$ (HIF $1 \alpha)$ were increased in co-treated cells compared with those treated with LPS only, and inhibiting OXPHOS or glutamine metabolism significantly decreased levels of both HIF1 $\alpha$ and IL- $1 \beta$ in co-treated cells. To investigate the metabolites to which HIF1a responds in co-treated cells, the authors looked at the metabolomic profile of LPS-stimulated macrophages with or without oxPAPC. Levels of oxaloacetate (OAA) were markedly increased in cells exposed to oxPAPC as a result of increased cytoplasmic metabolism of citrate. Administration of OAA to LPS-stimulated macrophages stabilized HIF1 $\alpha$ and increased IL-1 $\beta$ production, whereas inhibition of cytoplasmic citrate metabolism had the opposite effect.

Together, the results suggest that the metabolic changes of increased OXPHOS and glutaminolysis induced by oxPAPC culminate in increased production of OAA, which stabilizes HIF1 $\alpha$ and thus increases pro-IL-1 $\beta$ transcription (priming). Combined with the known effect of oxPAPC on inflammasome activation, this priming results in increased IL- $1 \beta$ production.

Of relevance to the in vivo situation, genetically hypercholesterolaemic mice fed a high-fat diet, which have therefore been pre-exposed to oxPAPC, had significantly increased plasma levels of IL- $1 \beta$ and increased mortality after LPS injection compared with wild-type mice. The hypercholesterolaemic mice could be protected from LPS-induced death by inhibiting glutamine metabolism or OAA production, and the same inhibitors also decreased IL- $1 \beta$ levels and the size of atherosclerotic plaques in these mice. Finally, the authors showed that in a cohort of 4,054 human peripheral blood samples, transcriptional signatures of OXPHOS, glutamine metabolism and OAA metabolism were significantly associated with having a proatherosclerotic lipid profile. Thus, in both mice and humans, exposure to oxidized phospholipids can drive a hypermetabolic, hyperinflammatory macrophage phenotype.

Kirsty Minton ORIGINAL ARTICLE Di Gioia, M. et al. Endogenous oxidized phospholipids reprogram cellular metabolism and boost hyperinflammation. Nat. Immunol. https://doi.org/10.1038/s41590019-0539-2 (2019) 